\title{
Pentoxifylline does not protect against hyperoxic lung injury in rats
}

\author{
E.T. Naureckas, P. Factor, O. Benjaminov, E. Hoffer, V. Sriram, J.I. Sznajder
}

Pentoxifylline does not protect against hyperoxic lung injury in rats. E.T. Naureckas, P. Factor, O. Benjaminov, E. Hoffer, V. Sriram, J.I. Sznajder. CERS Journals Ltd 1994. ABSTRACT: Hyperoxia has been used extensively as a model of acute lung injury. The drug pentoxifylline has been shown to have a protective effect in other models of lung injury. We sought to determine whether pentoxifylline protects against hyperoxic lung injury in rats by decreasing the accumulation of neutrophils within the lung.

A total of 84 rats were studied. Twenty four rats were randomized into four groups. Two groups of rats were pretreated for $48 \mathrm{~h}$ with either pentoxifylline (20 $\mathrm{mg} \cdot \mathrm{kg}^{-1}$ ) or saline, and then exposed to $>95 \% \mathrm{O}_{2}$ for $60 \mathrm{~h}$ while treatments continued. Two groups of control rats received the same treatment regimens as the $\mathrm{O}_{2}$-exposed animals, but breathed room air. Neutrophil accumulation in the lung was quantified both by histology and myeloperoxidase activity.

Lung neutrophil accumulation increased in the oxygen-exposed group receiving pentoxifylline as compared to oxygen- or air-exposed rats receiving saline injections. Total glutathione was higher in lung homogenates from the hyperoxic, pentoxifylline-treated group than in homogenates from the other three groups. To study survival, 60 rats were exposed to $>95 \% \mathrm{O}_{2}$ for $120 \mathrm{~h}, 30$ rats were pretreated with pentoxifylline, and 30 received saline. Survival after $120 \mathrm{~h}$ of exposure to hyperoxia was not altered by pentoxifylline treatment (pentoxifylline treated: 6 out of 30 survived; saline treated: 2 out of 30 survived).

We conclude that pentoxifylline does not reduce mortality or lung injury in rats exposed to hyperoxia and is associated with an increase in lung neutrophil accumulation.

Eur Respir J., 1994, 7, 1397-1402.

\begin{abstract}
Section of Pulmonary and Critical Care Medicine, Michael Reese Hospital, the University of Illinois Hospital at Chicago, the University of Chicago and the Technion, Israel Institute of Technology.
\end{abstract}

Correspondence: J.I. Sznajder Division of Pulmonary Medicine Michael Reese Hospital, RC 216 2929 S. Ellis Ave Chicago

IL 60616 USA

Keywords: Hyperoxia

lung glutathione

lung injury

lung neutrophils pentoxifylline

Received: December 161993 Accepted after revision May 21994

This study was supported in part by the MRIC of Michael Reese Hospital and the Winfield Fund. J.I.S. is a career investigator of the American Lung Association.
The toxic effects of hyperoxia upon the lungs are wellknown [1-3]. In adult rats, lung injury becomes evident after $50 \mathrm{~h}$, and death usually occurs within $72 \mathrm{~h}$ of exposure to $100 \%$ oxygen [1]. Neutrophils accumulate in the lungs of rats soon after exposure to $100 \%$ oxygen, and may play a role in this form of acute lung injury [4, 5].

Pentoxifylline, a methylxanthine, has been shown to be protective in models of acute lung injury due to sepsis or endotoxin, in part by preventing the sequestration of neutrophils within the pulmonary circulation $[6,7]$. It has been postulated that pentoxifylline inhibits the effect of tumour necrosis factor (TNF) on neutrophils [8-10], and/or inhibits monocyte synthesis of TNF in response to endotoxin [11]. Pentoxifylline exerts its effects on neutrophils at the cell surface through a mechanism that has yet to be characterized. SulLivan and coworkers [8] have shown that pentoxifylline restores TNF-inhibited neutrophil migration. Adenosine has been shown to exert a similar effect. Pentoxifylline, however, does not act through either of the known adenosine receptors, as specific antagonists of the $A_{1}$ and $A_{2}$ receptors do not block the effect of either adenosine or pentoxifylline [12].
In the present investigation, we tested whether intraperitoneal pentoxifylline affects lung injury and mortality by decreasing the accumulation of neutrophils in the lungs of hyperoxic rats. Lactate dehydrogenase (LDH) activity and albumin concentration in bronchoalveolar lavage fluid (BALF) were measured as indices of lung injury. Neutrophil accumulation in the lung was measured by histological examination and by myeloperoxidase assay. Total glutathione was also measured in lung tissue as an index of oxidative stress. Our results suggest that, in the rat hyperoxic model of lung injury, pentoxifylline does not reduce the accumulation of neutrophils within the lungs, nor does it protect against lung injury or decrease mortality.

\section{Materials and methods}

\section{Study animals}

Eighty four male 275-325 g Sprague-Dawley rats were used. All animal experiments conformed to the Helsinki convention for the use and care of animals. 


\section{Study design}

To determine whether pentoxifylline decreased hyperoxia-induced mortality, we pretreated rats for $48 \mathrm{~h}$ with intraperitoneal injections of either pentoxifylline or an equal volume of saline. The animals were then placed in a $>95 \% \mathrm{O}_{2}$ atmosphere, whilst treatment with either pentoxifylline or saline was continued. Mortality was determined at the end of $120 \mathrm{~h}$ of exposure.

To determine whether pentoxifylline reduced lung injury caused by sublethal oxygen exposure, we pretreated rats with either pentoxifylline or saline, as described above. The rats were then exposed to either air or $>95 \%$ $\mathrm{O}_{2}$ for $60 \mathrm{~h}$, before being sacrificed. This time interval was chosen as survival rate was almost $100 \%$ at $60 \mathrm{~h}$, in this model. Measurements of neutrophil accumulation within the lung were made using an assay of the enzyme myeloperoxidase, and by direct counting of histological sections. The lung tissue was also assayed for total glutathione as a index of oxidant injury. BALF was also obtained from each rat and assayed for cell count, albumin and lactate dehydrogenase (LDH).

\section{Mortality studies}

Sixty $275-325$ g male Sprague-Dawley rats were randomized into two groups. Thirty were pretreated for $48 \mathrm{~h}$ with pentoxifylline (generously supplied by HoechstRoussel) (20 mg. $\mathrm{kg}^{-1}$ b.i.d. by intraperitoneal (i.p.) injection). The other thirty were injected with an equivalent volume of normal saline. Both groups were placed in a $>95 \% \mathrm{O}_{2}$ environmental chamber (Kirchner Co.) and given food and water ad libitum, whilst injections of pentoxifylline or saline were continued. The number of deceased rats were counted every $12 \mathrm{~h}$ for up to $120 \mathrm{~h}$.

\section{Injury studies}

Twenty four male Sprague-Dawley rats were randomized into four groups. They received i.p. injections of $20 \mathrm{mg} \cdot \mathrm{kg}^{-1}$ of pentoxifylline or an equivalent volume of saline every $12 \mathrm{~h}$ for $48 \mathrm{~h}$. Eight rats receiving pentoxifylline injections and 8 rats receiving saline injections were then placed in a $>95 \% \mathrm{O}_{2}$ environmental chamber for $60 \mathrm{~h}$. The remaining 4 pentoxifylline-treated, and 4 saline-treated rats were maintained in room air. In all groups, treatment with pentoxifylline or saline was continued. The rats in all four groups were allowed food and water ad libitum. At the end of $60 \mathrm{~h}$, the rats were given a lethal injection of pentobarbital, heparinized, and exsanguinated by cannulation of the inferior vena cava. A sample of blood was saved for neutrophil isolation as described below. The heart and lungs of each rat were removed en bloc. The lungs were perfused with a phosphate buffered saline solution ( $\mathrm{pH} 7.4)$ containing $3 \mathrm{~g} \cdot \mathrm{dl}^{-1}$ albumin, at a pressure of $20 \mathrm{cmH}_{2} \mathrm{O}$. Perfusion was stopped when the fluid emerging from the cannula in the inferior vena cava was completely clear (approximately $20 \mathrm{ml}$ of perfusate). After ligation of the left main-stem bronchus, the right lung was slowly lavaged with $10 \mathrm{ml}$ of a $4^{\circ} \mathrm{C}$ saline solution, taking care not to exceed $15 \mathrm{cmH}_{2} \mathrm{O}$ of airway pressure. The lavage fluid was spun for cell count, and measurement of LDH and albumin. The lungs were dissected away from the heart, great vessels, and large airways, and portions of the left lung, which had not been subjected to BAL, were analysed for total glutathione (GSH), and myeloperoxidase activity, as described below.

\section{Myeloperoxidase assay}

To assess lung neutrophil number, myeloperoxidase (MPO) was measured using a modification of a method described previously [13]. In brief, $500 \mathrm{mg}$ samples of lung from each animal was subjected to three cycles of homogenization in $2.5 \mathrm{ml}$ of $50 \mathrm{mM}$ potassium phosphate buffer ( $\mathrm{pH} 6.0$ ) with $0.5 \%$ hexadecyltrimethylammonium bromide (HTAB), freezing in a methanol bath, and centrifugation at $2,000 \times \mathrm{g}(20 \mathrm{~min})$. The three supernatants were combined, passed through a $0.22 \mu \mathrm{m}$ millipore filter and assayed for myeloperoxidase activity. To normalize lung myeloperoxidase to neutrophil number, neutrophils from each rat were isolated from peripheral blood samples using Ficoll gradient sedimentation, followed by osmotic erythrocyte lysis. The neutrophil concentration was determined using a haemocytometer. The neutrophils were then suspended in $2.0 \mathrm{ml}$ of $0.5 \%$ HTAB/potassium phosphate buffer (50 mM, pH 6.0), subjected to three cycles of freezing in a methanol/dry ice bath, centrifuged at 2,000×g, and assayed for myeloperoxidase activity. A standard curve of myeloperoxidase activity versus neutrophil number was then determined for the neutrophils from each animal. A representative standard curve is shown in figure 1. MPO was assayed spectrophotometrically by adding a $50 \mu \mathrm{l}$ sample to 1.40 $\mathrm{ml}$ of $50 \mathrm{mM}$ phosphate buffer $(\mathrm{pH} 6.0)$ containing 0.167 $\mathrm{mg} \cdot \mathrm{ml}^{-1} \mathrm{o}$-dianisidine dihydrochloride. Hydrogen peroxide was added to bring the final concentration of $\mathrm{H}_{2} \mathrm{O}_{2}$ to $0.0005 \%$. The rate of change in adsorption was measured at $460 \mathrm{~nm}$ in a Beckman Model 35 spectrophotometer.

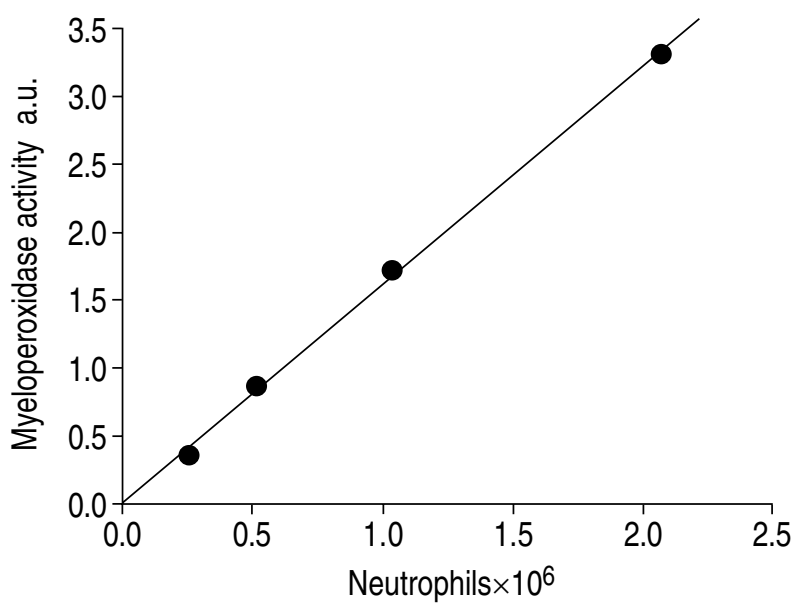

Fig. 1. - Representative standard curve of myeloperoxidase activity (arbitrary units (a.u.)) versus neutrophil number. 


\section{Neutrophil counts}

In order to independently verify the results of the myeloperoxidase assay, the right upper lobes from the rats in all four groups were fixed in formaldehyde and representative sections were prepared. The number of neutrophils contained in 10 alveoli was counted in 10 randomly chosen fields per rat by a pathologist (V.S.) blinded to treatment conditions.

\section{Glutathione assay}

Total glutathione was measured using a previously described protocol [14], where $0.25 \mathrm{~g}$ of lung was homogenized in $5 \mathrm{ml}$ of $10 \mathrm{mM}$ 5',5'-dithiobis 2-nitrobenzoic acid (DTNB), $5 \mathrm{mM}$ ethylene-diamine tetra-acetic acid (EDTA), $100 \mathrm{mM}$ potassium phosphate buffer, $\mathrm{pH} 7.4$ (Buffer A). Total glutathione was measured by adding $500 \mathrm{ml}$ of homogenate to $500 \mathrm{ml}$ of $10 \mathrm{mM}$ DTNB. The proteins were removed from the sample by precipitation with $500 \mathrm{ml}$ of $4 \%$ sulphosalicylic acid (SSA), followed by centrifugation at $2,000 \times \mathrm{g}$ for $5 \mathrm{~min}$. Fifty millilitres of the sample was then mixed in a cuvette with $50 \mathrm{ml}$ of DTNB solution $0.5 \mathrm{U}$ glutathione (oxidized form) (GSSG) reductase and $115 \mathrm{nmol}$ nicotinamide adenine dinucleotide phosphate (reduced form) (NADPH) in a $100 \mathrm{mM}$ potassium phosphate buffer $(\mathrm{pH} \mathrm{7.4)}$ to a total volume of $900 \mathrm{ml}$. The change in absorption at $412 \mathrm{~nm}$ with respect to time was then measured and referenced to a standard concentration of GSH.

\section{Bronchoalveolar lavage measurements}

To assess the effect of pentoxifylline on lung injury, BALF cell count (number $\cdot \mathrm{ml}^{-1}$ ) was determined by counting $10 \mathrm{ml}$ of BALF using a haemocytometer. All counts were made in quadruplicate. To assess capillary permeability, albumin was determined spectrophotometrically using Sigma (St. Louis, MO, USA) kit No. 625-2, and LDH was determined using Sigma assay No. dg 1340$\mathrm{K}$.

\section{Statistical analysis}

Results are expressed as mean \pm standard deviation. All group comparisons were made using one way analysis of variance (ANOVA), with a comparison of means by the Duncan method. The mortality comparisons were made by Chi-squared test.

\section{Results}

\section{Mortality study}

As shown in figure 2, pentoxifylline did not reduce mortality in rats exposed to hyperoxia. Six out of 30

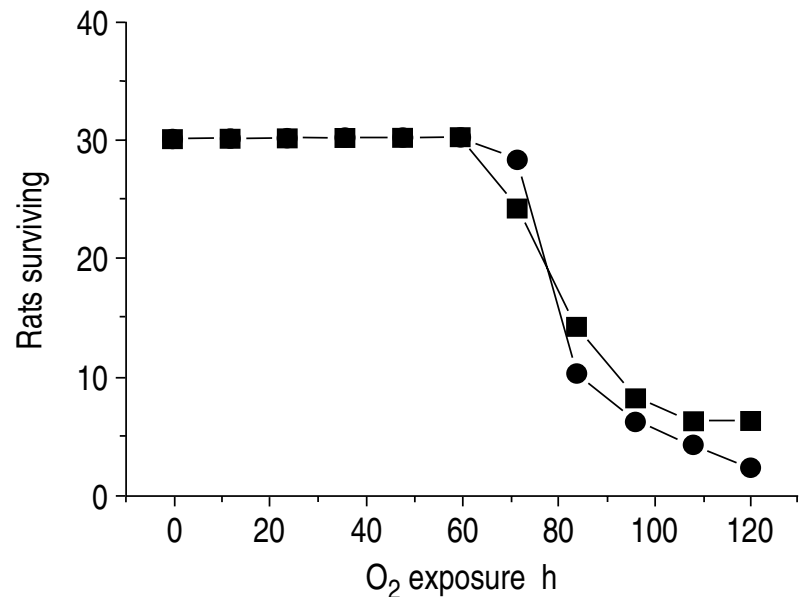

Fig. 2. - Mortality curve for rats exposed to hyperoxia. Hyperoxia was initiated at time $=0$ following $12 \mathrm{~h}$ of pretreatment with either pentoxifylline or saline. Survival was assessed every $12 \mathrm{~h}$. There was no significant difference between the two groups. $\mathbf{a}$ : pentoxifylline treated rats. ๑: saline treated rats

pentoxifylline-treated rats survived $120 \mathrm{~h}$ of $>95 \% \mathrm{O}_{2}$, whilst 2 out of 30 saline-treated rats survived the same challenge $(\mathrm{p}=0.10)$.

\section{Measurement of lung injury}

As shown in table 1, cellularity of the BALF of oxygen-exposed animals was significantly increased over the levels seen in normoxic animals $(\mathrm{p}<0.01)$. Pentoxifylline treatment did not independently change BALF cell count in either hyperoxic or normoxic animals. BALF albumin levels were elevated in the hyperoxiaexposed rats, whether receiving pentoxifylline or saline, over both normoxic groups $(\mathrm{p}<0.05)$, but were no different from one another. Levels of LDH in hyperoxic, pentoxifylline- or saline-treated rats, were greater than in either of the normoxic control groups $(\mathrm{p}<0.05)$, but were not different from each other.

As shown in figure 3, total GSH levels were higher in both oxygen-exposed groups when compared to the normoxic controls $(\mathrm{p}<0.05)$. Pentoxifylline treatment increased the GSH levels in hyperoxia-exposed rats over the levels seen in saline-treated rats exposed to hyperoxia $(\mathrm{p}<0.05)$. Total GSH levels did not differ between normoxic pentoxifylline-treated and normoxic salinetreated rats.

Table 1. - Effect of hyperoxia and pentoxifylline on cell count, albumin and LDH measured in bronchoalveolar lavage fluid

\begin{tabular}{lccc}
\hline Group & $\begin{array}{c}\text { Cell count } \\
\text { cells } \cdot \mathrm{ml}^{-1}\end{array}$ & $\begin{array}{c}\text { Albumin } \\
\mathrm{mg} \cdot \mathrm{dl}^{-1}\end{array}$ & $\begin{array}{c}\mathrm{LDH} \\
\mathrm{IU} \cdot \mathrm{l}^{-1}\end{array}$ \\
\hline Pentoxifylline/O & $34.0 \pm 8.6^{*}$ & $48.4 \pm 10.7^{+}$ & $29.9 \pm 6.1^{+}$ \\
Saline/O $_{2}$ & $45.9 \pm 7.2^{*}$ & $29.5 \pm 6.1^{+}$ & $23.6 \pm 3.7^{+}$ \\
Pentoxifylline/air & $3.3 \pm 2.2$ & $6.1 \pm 2.6$ & $5.9 \pm 2.9$ \\
Saline/air & $2.7 \pm 1.5$ & $13.5 \pm 9.9$ & $9.2 \pm 2.9$ \\
\hline
\end{tabular}

Data are presented as mean \pm standard deviation. +, *: difference $(p<0.05,<0.01)$ as compared to rats breathing room air; LDH: lactate dehydrogenase. 


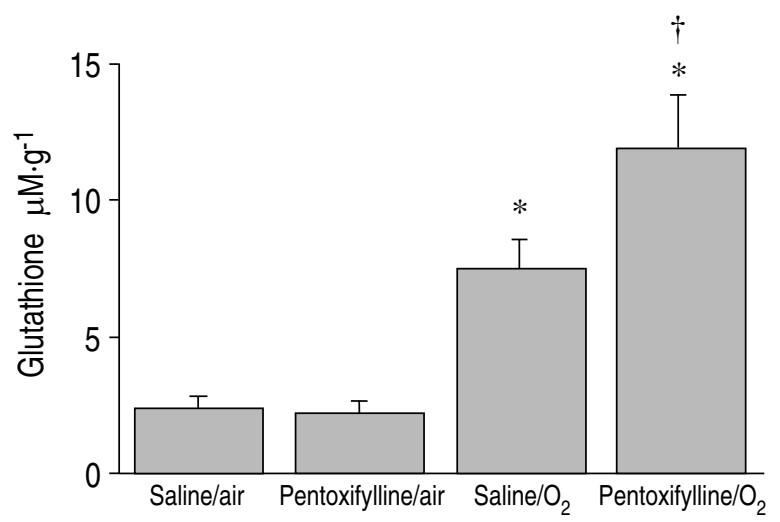

Fig. 3. - Total glutathione levels in rats pretreated with either saline or pentoxifylline and exposed to either room air or $>95 \% \mathrm{O}_{2}$ for $60 \mathrm{~h}$. Data are presented as mean \pm standard deviation. *: difference $(\mathrm{p}<0.05)$ as compared to room air-exposed rats; $\dagger$ : difference $(\mathrm{p}<0.05)$ as compared to room air and saline $/ \mathrm{O}_{2}$ animals.

\section{Neutrophil accumulation}

As assessed by myeloperoxidase activity (fig. 4a), the number of neutrophils was higher in hyperoxic animals receiving treatment with pentoxifylline than in those receiving saline treatment or saline-treated normoxic

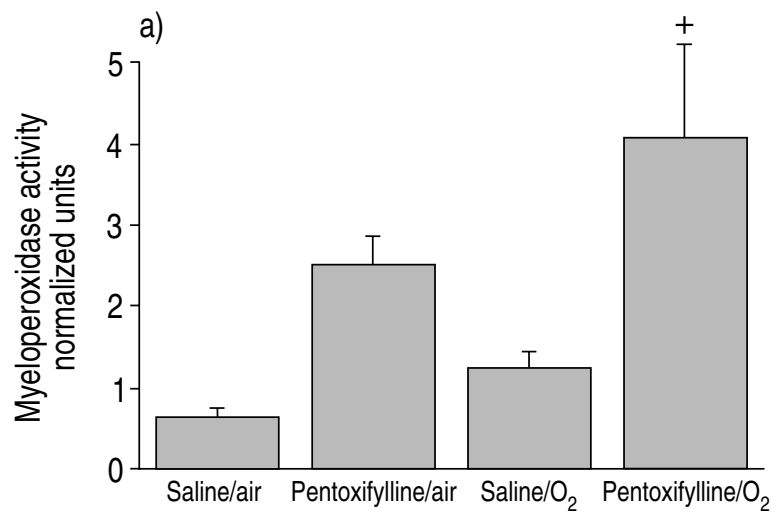
b)

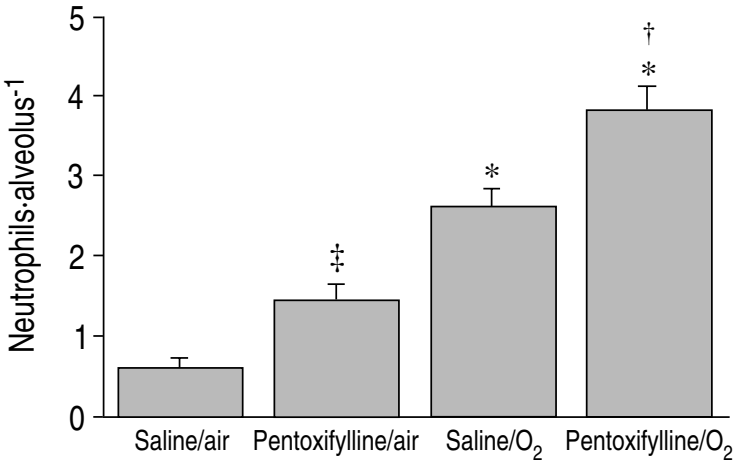

Fig. 4. - a) Effect of hyperoxia and pentoxifylline on neutrophil myeloperoxidase activity (neutrophils $\times 10^{6} \cdot \mathrm{g}^{-1}$ of lung tissue). Data are normalized to the myeloperoxidase activity of the circulating neutrophils for each rat and are presented as mean \pm standard deviation. + : difference $(\mathrm{p}<0.05)$ as compared to either saline $/ \mathrm{O}_{2}$ or room airexposed rats treated with saline. b) Effect of hyperoxia and pentoxifylline on the number of neutrophils counted per alveolus. Data are presented as mean \pm standard deviation. *: difference $(\mathrm{p}<0.01)$ as compared to room air-exposed groups; ${ }^{\dagger}$ : difference $(\mathrm{p}<0.05)$ with respect to hyperoxia-exposed, saline-treated rats; $\ddagger$ : $(\mathrm{p}<0.05)$ as compared to room air-exposed, saline-treated rats. animals $(\mathrm{p}<0.05)$. The number of neutrophils in the pentoxifylline-treated normoxic group tended to be higher but was not different from the normoxic, saline-treated rats $(\mathrm{p}=0.08)$.

The neutrophil numbers per alveoli, assessed by direct counting (fig. 4b), support the myeloperoxidase measurements. Hyperoxic rats treated with pentoxifylline had more lung neutrophils than saline-treated animals exposed to hyperoxia $(\mathrm{p}<0.05)$. Both saline- $\mathrm{O}_{2}$ and pentoxifylline- $\mathrm{O}_{2}$ rats had significantly greater numbers of neutrophils per alveolus than normoxic animals receiving either pentoxifylline or saline treatments $(p<0.01)$. Also, among room air-exposed rats, those treated with pentoxifylline had increased lung neutrophil accumulation $(\mathrm{p}<0.05)$.

\section{Discussion}

Hyperoxia has been used extensively as an animal model of acute lung injury. As the drug pentoxifylline has been found to be protective in other models of acute lung injury, we investigated whether it would ameliorate hyperoxia-induced lung injury and mortality in rats. We sought to determine whether pretreatment with this drug prevented the accumulation of neutrophils within the rat lung in our model, an effect well-documented in other lung injury models $[6,7]$.

We observed that pentoxifylline did not protect against hyperoxic toxicity in rats. Mortality was similar in the pentoxifylline- and saline-treated rats exposed to hyperoxia. The degree of lung injury as assessed by BALF cell counts, BALF LDH activity, and BALF albumin concentration, was not reduced by pentoxifylline treatment in hyperoxia-exposed rats. In contrast to the effect seen with pentoxifylline, in endotoxin-induced lung injury, we found an increase in the number of lung neutrophils in hyperoxic animals treated with pentoxifylline. In endotoxin-induced injury, pentoxifylline decreases the number of neutrophils within the lung, with a concomitant reduction in lung injury $[6,7]$. The contrasting responses to pentoxifylline in these two models of lung injury may be due to a difference in the role played by the neutrophil in each. In endotoxin-induced lung injury, damage is thought to be mediated through a number of cytokines, including TNF [15-17]. These agents act as potent activators of circulating neutrophils, inducing increased endothelial adhesion, degranulation and oxidative burst, as well as decreased chemotaxis [18, 19]. Evidence suggests that pentoxifylline protects against endotoxin-induced lung injury by counteracting these effects of TNF upon neutrophils [9-12, 20].

In hyperoxia-induced lung injury, a variety of cells, such as alveolar macrophages, produce chemotaxins. This results in the attraction and sequestration of neutrophils within the lung; neutrophils then produce lung injury by degranulation and free radical and proteolytic enzyme release [21, 22]. The lack of a protective effect of pentoxifylline in hyperoxic lung injury possibly suggests that TNF is not a major contributor in hyperoxia. Although neutrophils have been shown to contribute to 
lung injury in hyperoxia $[4,5]$, there is also data to suggest that hyperoxic lung injury may occur in the absence of neutrophils, as shown in a previous study, where neutrophil depletion by cyclophosphamide did not affect lung oedema in rats exposed to hyperoxia [23]. The production of free radicals has been shown to contribute significantly to hyperoxia-induced lung injury, and a number of studies have shown an improvement in survival with the use of free radical scavengers. WHITE $e t$ al. [24] demonstrated improved survival in rats pretreated with superoxide dismutase (SOD) and catalase (CAT) attached to polyethylene glycol. JACOBSON et al. [25] demonstrated a similar effect of conjugated SOD and CAT in a hyperoxic rabbit model. They also demonstrated a protective effect with vitamin $\mathrm{E}$ and hydroxianisol in this model.

Pentoxifylline has also been shown to counteract TNF inhibition of chemotaxis [12]. Sullivan and co-workers [17] reported enhancement of chemotaxis with pentoxifylline alone in the absence of TNF inhibition. The increase in accumulated neutrophils seen in the pentoxifylline-treated animals in our study may be explained by a greater migration of neutrophils into an area already damaged by free radical production. The accumulation of glutathione has been shown to be a marker of increased oxidative stress both in hyperoxic and endotoxic lung injury [13, 26-28]. In our study, pentoxifylline was associated with increased glutathione concentrations in hyperoxia-exposed rats. This effect may be due either to increased oxidative stress from the elevated number of neutrophils or to a direct, and yet unknown, effect of pentoxifylline.

There were a number of limitations of the study which may, in part, explain the negative results. If an insufficient dose of pentoxifylline were given, a higher dose of drug may result in a protective effect. However, when the doses used in our study were compared to other models showing a protective effect of pentoxifylline, this did not appear to be the case. A wide range of doses have been used in various models of lung injury. As the half life of intravenous pentoxifylline has been shown to be less than $2 \mathrm{~h}$ in humans [29], continuous i.v. infusions have been utilized by some investigators. The large numbers of animals required for this study and the difficulties inherent in continuous catheterization in rats made this choice impractical for this study. A number of studies have shown a protective effect of pentoxifylline with intermittent dosing. FLETCHER et al. [30] demonstrated that a single $20 \mathrm{mg} \cdot \mathrm{kg}^{-1}$ i.p. dose of pentoxifylline, administered $15 \mathrm{~min}$ after an intravenous lipopolysaccharide (LPS) infusion, significantly improved survival. In the same study, higher doses (100 $\mathrm{mg} \cdot \mathrm{kg}^{-1}$ i.p) resulted in decreased survival. An earlier study by CHALKIADAKIS et al. [31] demonstrated a protective effect by pentoxifylline in a rat peritonitis model. A dose of $17 \mathrm{mg} \cdot \mathrm{kg}^{-1}$ given intramuscularly once a day resulted in 2 deaths out of 20 rats, compared to 16 deaths out of 20 animals not receiving pentoxifylline pretreatment.

Another limitation of the study is that nonlabelled albumin is a relatively insensitive index of lung injury.
However, when considered together with the other indices of lung injury used, including BALF LDH and BALF cell count, the results indicate that pentoxifylline did not attenuate lung injury in this model.

In summary, this study shows that pentoxifylline treatment in rats does not reduce mortality or lung injury induced by hyperoxia. Pentoxifylline treatment does, however, increase neutrophil accumulation, possibly by increasing chemotaxis induced by factors other than TNF. This results in increased oxidative stress, as evidenced by elevated total glutathione in the pentoxifylline treated rats.

Acknowledgements: The authors would like to acknowledge the assistance of R. Samsel, in the preparation of this manuscript. They would also like to thank M. Fink for her assistance.

\section{References}

1. Jackson RM. Pulmonary oxygen toxicity. Chest 1985; 88: 900-905.

2. Klein J. Normobaric oxygen toxicity. Anesth Analg 1990; 70: 195-207.

3. Gholamhossain H, O'Neil JJ, Barry BE, Freeman BA, Crapo JD. Pulmonary injury in rats following continuous exposure to $60 \% \mathrm{O}_{2}$ for 7 days. J Appl Physiol: Respirat Environ Exercise Physiol 1981; 51(5): 12201231.

4. Barry BE, Crapo JD. Patterns of accumulation of platelets and neutrophils in rat lungs during exposure to $100 \%$ and $85 \%$ oxygen. Am Rev Respir Dis 1985; 132: 548555.

5. Rinaldo JE, English D, Levine J, Stiller R, Henson J. Increased intrapulmonary retention of radiolabeled neutrophils in early oxygen toxicity. Am Rev Respir Dis 1988; 137: 345-352.

6. Welsh CH, Lien D, Worthen GS, Weil JV. Pentoxifylline decreases endotoxin-induced pulmonary neutrophil sequestration and extravascular protein accumulation in the dog. Am Rev Respir Dis 1988; 138: 1106-1114.

7. Ishizaka $\mathrm{A}, \mathrm{Wu} \mathrm{Z}$, Stephens $\mathrm{KE}$, et al. Attenuation of acute lung injury in septic guinea-pigs by pentoxifylline. Am Rev Respir Dis 1988; 138: 376-382.

8. Sullivan GW, Carper HT, Novick WJ, Mandell GL. Inhibition of the inflammatory action of interleukin-1 and tumor necrosis factor (alpha) on neutrophil function by pentoxifylline. Infect Immunol 1988; 56: 1722-1729.

9. Lilly CM, Sandhu JS, Ishizaka A, et al. Pentoxifylline prevents tumor necrosis factor-induced lung injury. Am Rev Respir Dis 1989; 139: 1361-1368.

10. Zheng H, Crowley JJ, Chan JC, et al. Attenuation of tumor necrosis factor induced endothelial cell cytotoxicity and neutrophil chemoluminescence. Am Rev Respir Dis 1990; 142: 1073-1078.

11. Schandene L, Vandenbussche $\mathrm{P}$, Crusiaux A, et al. Differential effects of pentoxifylline on the production of tumor necrosis factor-alpha and interleukin- 6 by monocytes and T-cells. Immunology 1992; 76: 30-34.

12. Sullivan GW, Linden J, Hewlitt EL, Carper HT, Hylton JB, Mandell GL. Adenosine and related compounds counteract tumor necrosis factor-alpha inhibition of neutrophil migration: implication of a novel cyclic AMP-independent action on the cell surface. J Immunol 1990; 145: 1537-1544. 
13. Goldblum $\mathrm{SE}, \mathrm{Wu} \mathrm{K}$, Jay $\mathrm{M}$. Lung myeloperoxidase as a measure of pulmonary leukostasis in rabbits. $J$ Appl Physiol: Respirat Environ Exercise Physiol 1985; 59: 1978-1985.

14. Chang S, Lauterburg BH, Voelkel NF. Endotoxin causes neutrophil independent stress in rats. J Appl Physiol 1988; 65: 358-367.

15. Mathison JC, Wolfson E, Ulevitch RJ. Participation of tumor necrosis factor in the mediation of Gram-negative bacterial lipopolysaccharide-induced injury in rabbits. J Clin Invest 1988; 81: 1925-1937.

16. Tracey KJ, Beutler B, Lowery SF. Shock and tissue injury induced by recombinant human cachectin. Science 1986; 234: 470-474.

17. Stephens KE, Ishizaka A, Larrick JW, Raffin TA. Tumor necrosis factor causes increased pulmonary permeability and edema: comparison to septic acute lung injury. Am Rev Respir Dis 1988; 137: 1364-1370.

18. Salyer JL, Bohnsack JF, Knape WA, Shigeoka AO, Ashwood ER, Hill HR. Mechanisms of tumor necrosis factor-alpha alteration of PMN adhesion and migration. Am J Pathol 1990; 136: 831-841.

19. Ferrante A, Nandoskar A, Waltz D, Kowanko G. Effects of tumor necrosis factor-alpha and interleukin-1 alpha and beta on human neutrophil migration, respiratory burst and degranulation. Int Arch Allergy Appl Immunol 1988; 86: 82.

20. Sullivan GW, Patselas TN, Redick JA, Mandel GL. Enhancement of chemotaxis and protection of mice from infection. Trans Assoc Am Phys 1984; 97: 337-345.

21. Harada RN, Vatter AE, Repine JE. Macrophage effector function in pulmonary oxygen toxicity: hyperoxia damages and stimulates alveolar macrophages to make and release chemotaxins for polymorphonuclear leukocytes. J Leukocyte Biol 1984; 35: 373-383.

22. Fox RB, Hoidal JR, Brown DM, Repine JE. Pulmonary inflammation due to oxygen toxicity: involvement of chemotactic factors and polymorphonuclear leukocytes. Am Rev Respir Dis 1981; 123: 521-523.

23. Lurie A, Theven D, Braun-Pascund M, Fay M, Pocidalo JJ. Acute hyperoxia is not reduced by granulocyte depletion in rats. Respiration 1988; 53: 232-238.

24. White CW, Jackson JH, Abuchowski A, et al. Polyethylene glycol-attached antioxidant enzymes decrease pulmonary oxygen toxicity in rats. J Appl Physiol 1989; 66: 584-590.

25. Jacobson JM, Michael JR, Jafri MH, Gurtner GH. Antioxidants and antioxidant enzymes protect against pulmonary oxygen toxicity in the rabbit. $J$ Appl Physiol 1990; 68: 1252-1259.

26. Beehler CJ, Simchuck ML, Toth KM, et al. Blood sulfhydryl levels increase during hyperoxia: a marker of oxidant lung injury. J Appl Physiol 1989; 67: 10701075.

27. White CEW, Mimmack RF, Repine JE. Accumulation of lung tissue oxidized glutathione (GSSG) as a marker of oxidant-induced lung injury. Chest 1986; 89: $111 \mathrm{~S}-113 \mathrm{~S}$.

28. Jenkinson SG, Black RD, Lawrence RA. Glutathione concentrations in rat lung bronchoalveolar lavage fluid: effects of hyperoxia. J Lab Clin Med 1988; 112: 345-351.

29. Rames A, Poirier JM, Lecoz F, et al. Pharmokinetics of intravenous and oral pentoxifylline in heathy volunteers and in cirrhotic patients. Clin Pharmacol Ther 1990; 47: 354-359.

30. Fletcher MA, McKenna TM, Owens EH, Nadkarni VM. Effects of in vivo pentoxifylline treatment on survival and ex vivo vascular contractility in a rat lipopolysaccharide shock. Circ Shock 1992; 36(1): 74-80.

31. Chalkiadakis GE, Kostakis A, Karayannacos PE, et al. Pentoxifylline in the treatment of experimental peritonitis in rats. Arch Surg 1985; 120: 1141-1144. 